\title{
Psychological Interventions in Pain Management
}

\author{
Mustafa Nadeem Kirmani ${ }^{1}$, Firdos Jehan ${ }^{2}$, Rumana Sanam ${ }^{3}$
}

\begin{abstract}
Pain is among the most common somatic complaints. Fortunately, in only a minority of people is pain long lasting and severe, such that it interferes with daily life activities. Those with chronic, disabling pain present to healthcare providers repeatedly. Often they experience anxiety and depression, irritation, frustration and helplessness, and they suffer from insomnia and excessive medication use. It results from complex interplay of biological, psychological \& sociocultural factors. There are gamut of medical and psychosocial factors which cause pain. Pain is basically a sensory, emotional and subjective experience. High psychological arousal and cognitive set are significant factors which maintain or exacerbate the pain. In this paper, psychological strategies of pain management are being discussed. The major focus being cognitive behavioral interventions. The paper focuses on psychological factors related to pain and the role of professional psychologists in dealing with cases of chronic or other pain related disorders. The paper highlights the importance of incorporating biopsychosocial model in pain management for speedy recovery and better quality of life of pain patient.
\end{abstract}

Keywords: Pain, psychological management, biopsychosocial model

Pain is a complex, personal, subjective and unpleasant sensory and perceptual experience that may or may not have any correlation with bodily injury or tissue damage. The International Association for the study of pain defines pain as "Sensory and emotional experience" (IASP, 1979). Emotional processes are central to the experience and expression of pain. Pain is the outcome of a complex interplay of influences, including psychological factors, which may operate both as risk factors in and consequences of pain. Psychological factors play an important role in the onset, severity, exacerbation or maintenance of the pain. During the past half century, psychological thought has moved away from linear to multicausal models of pain (Gamsa, 1994). When a psychological causation of pain is postulated, multiple determinants of pain are usually also discussed.

Pain is among the most common somatic complaints. Fortunately, in only a minority of people is pain long lasting and severe, such that it interferes with daily life activities. Those with chronic, disabling pain present to healthcare providers repeatedly.

\footnotetext{
${ }^{1}$ Research Scholar, Department of Psychology, Aligarh Muslim University (AMU), Aligarh

${ }^{2}$ Rehabilation Psychologist, Aligarh, U.P

${ }^{3}$ Post graduate (M.A) Student, Department of Psychology, AMU, Aligarh

(C) 2015 I M Kirmani, F Jehan, R Sanam; licensee IJIP. This is an Open Access Research distributed under the terms of the Creative Commons Attribution License (http://creativecommons.org/licenses/by/2.0), which permits unrestricted use, distribution, and reproduction in any Medium, provided the original work is properly cited.
} 
Often they experience anxiety and depression, irritation, frustration and helplessness, and they suffer from insomnia and excessive medication use. It is well known that this group of chronic sufferers is difficult to treat: there is no immediate and definitive solution available for their pain problem. Great progress has been made in our understanding of the therapeutic strategies with different agents and techniques on pain in the past decades, but the analgesic result is not as effective as in chronic pain. Two hundreds years ago, the word "placebo" was defined by Robert Hooper in his dictionary to a more modern medical meaning as "any medicine adapted more to please than benefit the patient" (Hooper, 1811).

Evidence has accumulated that psychological factors play a major role in the development and maintenance of many features of a pain in clients presenting for treatment, largely independent of the original cause of their pain. The level of their disability is associated with more emotional distress and maladaptive conditions than with pathophysiology. Many of their problem are the result of inappropriate learning behaviours in dealing with pain and its consequences, for example pain complaints, resting, limping and taking medication. These behaviours are reinforced by their consequences or by the contingencies existing in the client's current environment. Cognitive and emotional factors are also involved, fear, anxiety, attributions, beliefs, self-efficacy, sense of having control over pain, and coping strategies interact in complex ways in the development of the problem of pain. Signs of emotional distress are frequently the most clearly recognizable evidence that another person is experiencing pain. To communicate their emotional distress most people readily offer dramatic affective language, describing pain in terms of tension, fear and autonomic distress. Expressions such as terrifying, frustration are often accompanied by paralinguistic vocalization of meaning and groaning and non-verbal signs of affective discomfort to signal the sufferer's distress to others. The most common emotional concomitants of pain are anxiety, fear and depression. Pain also involves gamut of emotional states like anger aggression and guilt. Pain also creates fear of avoidance whereby anxiety about activities exacerbating the pain stimulates the avoidance of those activities. Activity patterns and daily routines become disrupted while depression and catastrophizing thoughts are obstacles to recovery. Pain behaviours like groaning, crying etc. are reinforced by the social response they bring (attention, sympathy, legitimization of the behavior, and a temporary reduction of pain). Passive behaviours such as resting, reading, or watching television, which may be pleasant and associated with less pain, may become predominant. Pain behaviours may also buy time out of undesirable duties or activities.

\section{Psychological Analgesia}

It is a broad concept that includes all aspects referring to the psychological intervention. Hypnosis, music therapy, preoperative education, and linguistic suggestion all belong to psychological approaches in pain control. Whatever psychological methods used in analgesia, common neurophysiological mechanisms exist and different models explain its function. Functional magnetic resonance imaging (fMRI) verified an increase in neural activity during placebo associated psychological stimulation that is related to two major pain modulation 
mechanisms : i) affective regulation which includes activation of the rostral anterior cingulate cortex, bilateral amygdala, and medial prefrontal cortex; and ii) higher cognitive regulation during which the posterior cingulate, pre-cuneus, rostral anterior cingulate cortex, perihippocampal gyrus, and the temporal lobes are activated. As the "gate theory" described that afferent inhibition blocks ascending signals from the periphery, psychological stimuli at the early period produce analgesic effect through a self reinforcing feedback mechanism.

Several models give an in-depth understanding of the psychological stimulation associated analgesia. Conditioning, expectancy, motivation, and emotion are four psychological mediators involving in the process of analgesia. Conditioning model says that the interventional effect presented when the individual without knowing the stimulation would be and this process would not produce cognition. In this model,the perception of pain after psychological treatment largely depends on the learning history of the individual which determines the response variability under different context. The psychological conditioning as well as the verbal suggestion can turn tactile stimuli into pain and low-intensity pain into high-intensity pain. For this, the direct evidence was the conditioned pain reduction could be absolutely removed when the psychological stimuli were explained (Montgomery \& Kirsch, 1997). Originally, conditioning is the primary response to psychological analgesia. Following conditioning, expectancy of the psychological stimuli to produce an effective analgesia takes place. Once the patients expect to have an improvement in pain management, the effect of psychological analgesia would play its role. Due to anxiety and fear to pain, patients generally want to have rapid and effective methods that can relieve their pain which consequently leads to an expectancy of their pain therapies. Under this condition, physicians' attitude and enthusiasm takes an important part in whether or not the psychological analgesia comes into play (Weintraub, 2005). Give patients the hope to conquer pain accompanying with a warmth care, the expected effect of psychological analgesia would be maximized. After expectancy, motivation of analgesia is another aspect in determining the effect of psychological interventions. If the patient desires for a relief of pain, the real analgesic role of psychological stimuli would be magnitude. The motivation itself whether or not could predict the psychological effect on one type of pain needs to be explored at length, and could it be effective for Psychological Strategies in Pain Management. A body of literature has confirmed the role of emotions in pain perception and alleviation. Anxiety and stress are two main factors of emotionassociated psychological mediator. It is believed that anxiety is the cause of increased levels of pain, and reduction in anxiety produces analgesia (Luciano et al., 2011).Stress sometimes is related with increased levels of pain, but in some contexts, stress can produce analgesia (Donello et al., 2011). Therefore, the purpose of psychological suggestion in pain control is to alleviate patients' anxiety and stress, which in turn produces a feedback analgesia effect

\section{The Operant Model}

In developing and providing the initial tests of the operant model of $\mathrm{CP}$, Fordyce and his colleagues (Fordyce et al.,1968) gave birth to what became the field of psychological pain management. Operant theory hypothesizes that all behavior is sensitive to the effects of 
environmental responses to that behavior. Behaviors followed by reinforcers will maintain or increase in frequency, and behaviors that are ignored or punished may briefly increase but will ultimately decrease in frequency. Fordyce noted that "pain behaviors" - the things that people do that communicate pain to others (i.e., overt expressions of pain and suffering such as limping and grimacing) - are no different than any other behavior with respect to their sensitivity to environmental influences. Pain behaviors followed by reinforcing events, such as affection or sanctioned time out from social responsibilities, will increase in frequency. However, if pain behaviors are systematically ignored, and behaviors incompatible with them-so-called "well behaviors" such as exercise and maintaining an active lifestyle, including employment-are encouraged or positively reinforced,then over time these well behaviors will increase and pain behaviors will decrease. Interest in relaxation training as a treatment for pain continues, and relaxation training is often a component of interdisciplinary pain treatment and cognitivebehavioral therapy regimens. Relaxation training is also sometimes used as a stand-alone treatment for tension-type headaches. However, there is an increasing recognition that the beneficial effects of relaxation training alone on pain tend to be limited.

\section{Cognitive and Coping Models}

During the 1970s and early 1980s, the field of psychology expanded to include a greater interest in cognitions (e.g.,beliefs, attributions, expectations) and cognitive treatments (Beck, 1979; Dember, 1974;Lazarus \& Folkman, 1984; Meichenbaum, 1976), and psychologists began to apply cognitive models to the problem of CP (Turk et al., 1983).Cognitive models argue that our understanding of and ability to predict and influence behavior and mood are enhanced when we take into account an individual's beliefs, attributions, motivation, and intentions. These factors are reflected in an individual's thoughts, and thought processes represent viable treatment targets. Perhaps the most common intervention to emerge from this time period was cognitive therapy, which consists of teaching the patient to be aware of his or her thoughts and to evaluate those thoughts with respect to their overall rationality and helpfulness (vs. their irrationality and unhelpfulness). As treatment progresses, patients are encouraged to develop and focus on adaptive thoughts when irrational or unhelpful thoughts are identified.The primary assumptions shared by all CBT interventions are as follows: (a) People are active processors of information; (b) people are capable of gaining control over their thoughts, feelings, behaviors, and to some extent physiological processes; and (c) there are interrelationships among thoughts, feelings, behaviors, and physiological processes, and changes in one or more of these factors may result in changes in the others. Moreover, with CBT there is an emphasis on or at least an inclusion of components that target maladaptive cognitive content or processes. Finally, all CBT approaches emphasize patients'self-management of their lives coupled with optimism about peoples' abilities to function even when experiencing symptoms such as pain or fatigue. Despite the tactical problem related to differences in the specific therapeutic elements of CBT interventions, research supports the efficacy of CBT interventions for reducing pain and improving physical and psychological functioning in adults and children with persistent pain, at least modestly and to a degree comparable to that for other treatments ( Eccleston, \& Morley, 2009).The list of CP 
interventions based on cognitive and coping models (i.e., CBT interventions) is large and appears to be growing. It includes coping skills training (e.g., Keefe et al., 1999), mindfulness-based stress reduction ( Schmidt et al., 2011), motivational interviewing, acceptance-based interventions and graded exposure in vivo.Although a number of different treatments can be classified as CBT interventions, each has a different emphasis. For example, cognitive therapy focuses on teaching patients to monitor and evaluate their thoughts with respect to how adaptive (e.g., reassuring, calming) or maladaptive (e.g., alarming, distressing) they are, to facilitate the adaptive cognitions, and to replace maladaptive cognitions with more adaptive ones. Motivational interviewing targets "change talk" that is hypothesized to increase the probability of the use of coping responses thought to be adaptive and to decrease the probability of the use of coping responses thought to be maladaptive.

\section{BIOPSYCHOSOCIAL MODEL}

Engel (1977) gave 'Biopsychosocial' approach to medicine. The biopsychosocial model presumes some form of physical pathology or at least physical changes in the muslces, joints, or nerves that generate nonnoceptive input to the brain. At the periphery, nonoceptive factors transmit sensation that may or may not be interpreted as pain. Such sensation is not yet considered pain until subjected to higher order psychological and mental processing that involve perception, appraisal and behavior. Perception involves the interpretation of nouceptive input and identifies the type of pain (sharp, burning). Appraisal involves the meaning that is attributed to the pain and influences subsequent behaviors. The biopsycholgoical model has been instrumental in the development of cognitive behavioral treatment approaches for chronic pain, including assessment and intervention.

\section{Classification of Pain}

(1) Acute Pain : Pain that persists for less than 6 months is called acute pain. It is self-limiting and usually remits of its own without the need of treatment by a healthcare professional.

(2) Chronic pain: Pain that lasts for more than 6 months is called chronic pain. eg. Onteoarthritis, Fibromyolgia, etc.

(3) Recurrent Acute Pain : Pain that is characterized by pain episodes that alternate with pain free periods often in an unpredictable fashion is called recurrent acute pain.

\section{Epidemiology of Pain}

In the USA, there may be over 30 million people with chronic pain. Data obtained from the American Survey of Pain specialists suggest that approximately 2.9 million $(1.1 \%)$ of the population are treated annually by Healthcare professionals specialized in chronic pain (Market data Enterprise, 1995). 


\section{Psychology of Pain}

Emotional distress is common in people with chronic pain. Another pain persists, they may be unable to work, have financial difficulties, difficulty in performing everyday activities, sleep disturbance, or treatment related complications. They may be fearful and have inadequate or maladaptive support systems and other coping resources available to them. These consequences of chronic pain can result in depression, frustration, irritation, anger, anxiety, self-preoccupation or isolation. They feel totally demoralize. Biomedical factors appear to instigate the initial report of pain. Overtime, however, psychosocial and behavioural factors may serve to maintain and exacerbate the level of pain, influence adjustment and contribute to excessive disability. From this view, pain that persists over time should not be viewed as solely physical or purely psychological, the experience of pain is maintained by an interdependent set of biomedical, psychosocial and behavioural factors.

Health care providers need to consider not only the physical basis of pain but also clients' mood, fears, expectancies, coping resources and the response of significant others, including themselves. Regardless of whether there is an identifiable physical basis for the reported pain, both psychosocial and behavioural factors will interact to influence the nature, severity and persistence of pain and disability.

In order to understand the psychology of pain better, some specific affective and cognitive factor, that are particularly important in understanding chronic pain sufferer, and their experiences are now discussed.

\section{(1) Affective/Emotional Factors}

Pain is ultimately a subtective, private experience which is invariably described in terms of sensory and affective properties. The affective components of pain include negative emotions especially depression, anxiety and anger.

\section{(a) Depression}

Studies suggest that 40-50\% of chronic pain clients suffer from significant depression (Banks and Kerns, 1996). Turks (1995) determined that individual's appraisal of the effects of pain on their lines and of their ability to exert control over the pain and their lives mediated the paindepression relationship. These clients who believed that they could continue to function and maintain control despite their pain did not become depressed.

\section{(b) Anxiety}

Anxiety is also one of the prevalent emotions observed in chronic pain clients. In the absence of physical pathology to explain their pain they become fearful. The fear relates to activities that they anticipate will increase their pain. These fears may contribute to inactivity and greater disability. In addition to fear of movement, people with chronic pain may be anxious about the meaning of their symptoms for the future will their pain increase, will their physical capacity diminish, will they have progressive disability and ultimately end up in a wheel-chair or bedridden? In addition to those sources of fear, pain sufferers may fear that on the one hand people 
will not believe that they are suffering and on the others they may be told they are beyond help and just have to learn to live with it. All of these fears will contribute to increase muscle tension and physiological arousal that may exacerbate and maintain pain.

\section{(c) Anger}

Anger has been widely observed in people with chronic pain. 55\% of clients reported "bottled up anger". Kern,Rosenberg and Jacob (1994) noted that the internalization of angry feelings was related to measures of pain intensity, perceived interference and reported frequency of pain behaviours. Anger and hostility tend to increase the severity of pain. It is thus reasonable to expect that the presence of anger may serve as a complicating factor, increasing autonomic arousal and blocking motivation and acceptance of treatments oriented toward rehabilitation and disability management rather than cure. It is important to be aware of the significant role of negative mood on chronic pain clients because it effects treatment motivation and adherence to treatment recommendation.

\section{(2)Cognitive Factors}

Cognition refers to beliefs, attitude, perception, knowledge and appraisal of any phenomenon. The term appraisal has come from the psychology of emotion. Appraisal means one's interpretation and understanding of a particular situation or one's own somatic/bodily processes. Cognitive factors are determined by one's indirect or direct experiences. Cognitions are either directly or indirectly related to pain perception. They either precipitate or maintain or exacerbate pain. Cognitive behavioral interventions for pain management work on modifying dysfunctional beliefs related to pain and help building effective coping skills and through healthy coping statements.

\section{Chronic Pain and Cognitive Behavioral Interventions}

Many medical conditions like osteoarthritis, pain associated with oncology issues, fibromyalgia and gamut of medical conditions lead to chronic pain in the patients. Chronic pain is not only one of the highly prevalent problem but also a costly issue for people suffering from it, health care system and society in general. Patients with chronic pain report impairments of multiple qualityof-life measures, including physical, social and psychological well-being. This mixture of physical, emotional and social factors often complicates managing patients with chronic pain. Treatment of chronic pain needs to address the physical pathology that initiated the chronic pain, as well as the important social and psychological sequelae of chronic symptoms. Although multiple medical, surgical and other physical interventions are available, patients with chronic pain continue to experience symptoms with significant distress and disability. As a consequence, patients experience frustration, emotional distress, feelings of helplessness, and an overall sense of demoralization as they continue their quest to achieve relief. Psychological treatments are often considered when medical interventions prove to be inadequate but often are not integrated with traditional medical approaches. Psychological approaches used alone or in combination 
with appropriate pharmacological strategies, should be an integral part of care plans for most chronic pain patients. Psychological interventions used in combination with appropriate drug regimen often improve overall pain management, enhancing therapeutic effects while allowing reduction of medication doses to prevent or diminish adverse drug effects. Based on empirical research, there are three most common types of psychological treatment of chronic pain include:

1. Cognitive Behavioral Therapy (CBT),

2. Relaxation Training, and

3. Biofeedback.

These approaches are often used together to provide simultaneous interventions at cognitive and physical levels. Research on cognitive behavioral interventions in chronic pain involves CBT, relaxation therapy, biofeedback, or some combination of the three. Generally, some form of CBT is combined with either relaxation training or biofeedback.

\section{ASSESSMENT}

Patients with chronic pain need to feel understood by those who are providing care to them. On the other hand, a therapist requires relevant and adequate information about the patient from a bio-psychosocial perspective to establish therapeutic goals. Therefore, a comprehensive psychological assessment is a prerequisite for CBT and other interventions. Before starting the therapy clinician should have understanding of the following:

1. The patient in his or her physical and social environment,

2. The patient's relevant strengths and weaknesses,

3. The evidence for any psychopathology,

4. The nature of the disease and treatment regimen, and

5. The coping skills being used by the patient,

\section{Cognitive Behavioral Therapy}

CBT uses active, structured techniques aimed at modifying thoughts and behaviors and assisting individuals in developing a perspective of personal control and self-management of their pain. The components of CBT include

- Reconceptualization of the pain experience as subject to personal control

- Identification of idiosyncratic beliefs about pain and pain treatment; through the influence of thoughts, feelings, and physical activities

- Training in a number of cognitive and behavioral coping skills and presentation and discussion of their rationale

- $\quad$ Practice and consolidation of these coping skills through imagery, rehearsal, role playing, and contingent reinforcement of their appropriate use. 


\section{Figure 1}

Summary of Cognitive-Behavioral Therapy (CBT) Techniques

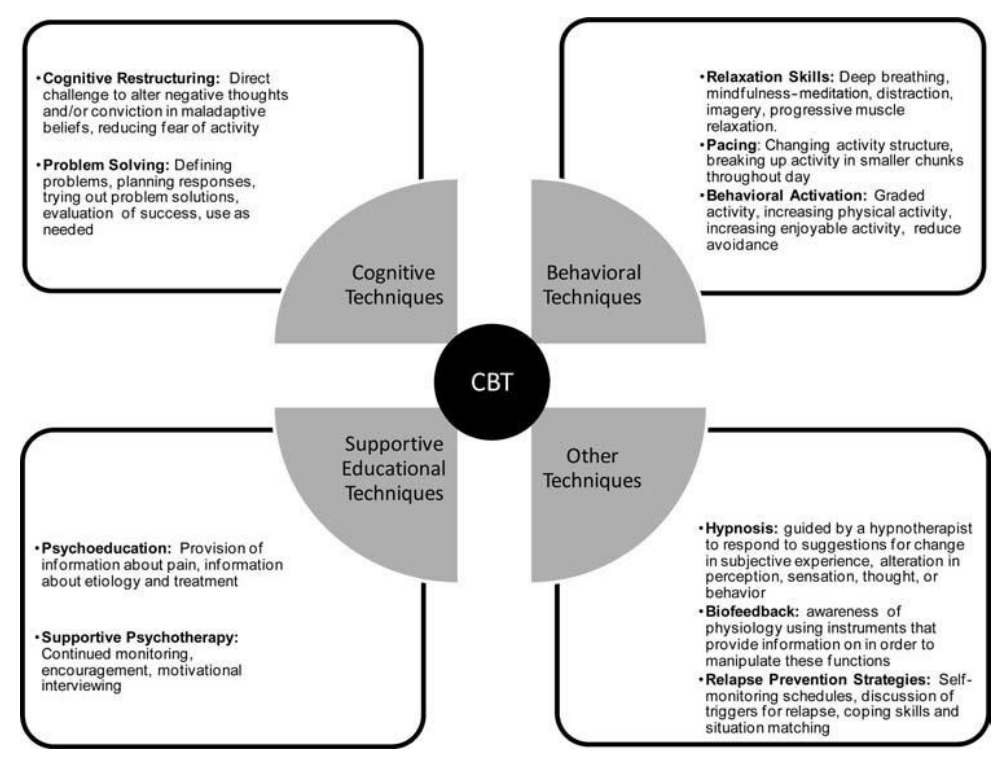

Note. From "Cognitive-Behavioral Perspective and Cognitive-Behavioral Therapy for People With Chronic Pain: Distinctions, Outcomes, and Innovations" by M.Skinner, H. D. Wilson, and D. C. Turk, 2012, Journal of Cognitive Psychotherapy, 26, p. 98. Copyright 2012 by Springer Publishing Company.

\section{Patient Education and Preparation for the Cognitive Behavioral Therapy}

The importance of patient education cannot be overemphasized. Such education programs commonly include information about the nature of pain and how to use pain assessment instruments, medications, and non-pharmacological pain management strategies. For many patients, especially older persons, family caregiver education is also essential. Whether the program is conducted one-on-one or organized in groups, it should be tailored to patients' needs and levels of understanding. The use of suitable written materials and appropriate methods for reinforcement is important to the success of the program.

Introducing the cognitive behavioral model into treatment requires some preparation of the patient. Preparing the patient for CBT involves reconceptualization of pain experience and establishing a collaborative therapeutic relationship. It can begin with dispelling the myth or misconception that the patient has been referred to a mental health professional because the pain is "in your head". One must always be careful not to imply that "the pain is all in our head" or not believable. It is helpful to present therapeutic techniques as methods to help the person manage the pain more effectively, improve the quality of life, and improve one's mood even though the pain will still be there. Patients with chronic pain need to understand and accept that one of the main aims of CBT is to enable them to develop more effective coping strategies. 
Explaining and discussing the gate control theory of pain with the chronic pain model along with examples form the foundation upon which the multifaceted intervention will be justified to the patient. Patients can be encouraged to explore links between cognitive behavioural factors and their pain. Active participation of patient in therapeutic program is essential for its success. It is helpful to explain that psychological pain management techniques differ from the many medical treatments the patient has likely undergone. In contrast to medical treatments, the patient must be actively involved in cognitive behavioural intervention program. Giving the rationale behind the various interventions (e.g., cognitive behavioral strategies, relaxation training, etc) to the patient also helps in the promotion of reconceptualization of pain experience as well as in his or her active involvement in the therapy.

\section{COGNITIVE RESTRUCTURING}

The cognitive behavior therapist and patient work together to identify specific patterns of thinking and behavior that underpin the patient's difficulties. Treatment continues between sessions with homework assignments both to monitor and challenge specific thinking patterns and to implement behavioural change. The cognitive methods in therapy include:

- Detailed explanation and discussion of the cognitive model to develop understanding about connections between thoughts, affect, and behavior using ABCDE paradigm

- Keeping a diary monitoring situations, thoughts, and feelings to develop awareness about these (Box 1).

- Identifying connections between thoughts, affect, and behavior

- Identifying specific cognitive errors or distortions

- Examining evidence "for" and "against" the thoughts

- Coaching patients in challenging negative thoughts by questions and other rational techniques

- $\quad$ Learning to identify dysfunctional assumptions underpinning distortions

- $\quad$ Cognitive rehearsal of coping with difficult situations or use of imagery 


\section{Box 1: Thought Diary}

Date :

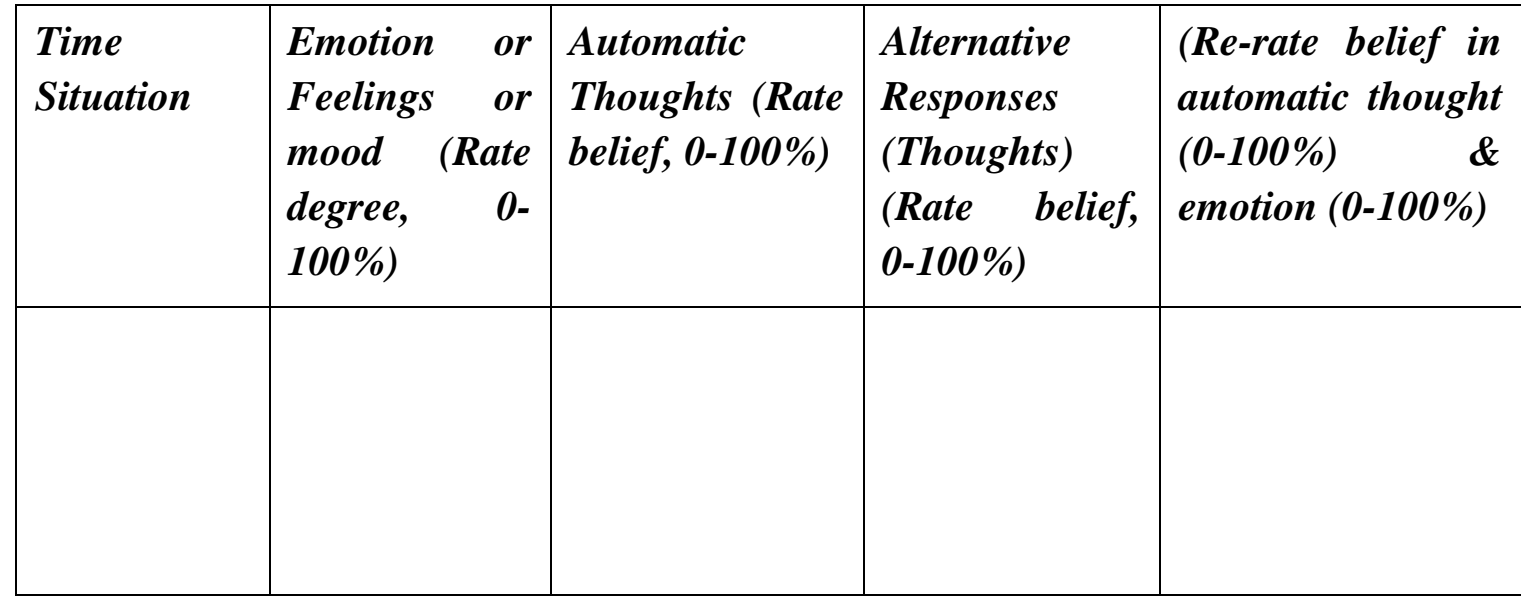

Challenging Dysfunctional Automatic Thoughts: Following key questions can be utilized to challenge dysfunctional or negative automatic thoughts identified:

- Is there an alternative explanation?

- What is the evidence that this thought is true ?

- What are the advantages and disadvantages of thinking this way?

- What is the best outcome, worst outcome, and most realistic outcome?

- What is the likelihood that this will happen?

- Am I asking questions that have no answer?

Patients are helped to learn application of these key questions to their own negative thoughts whenever they occur. They can select one or two questions that are useful in identifying the underlying logical problems in their thinking.

\section{Reducing Catastrophizing and Promoting Appropriate Coping Strategies}

Catastrophizing is one of the most common cognitive errors observed in chronic pain patients; therefore, it should be identified and specifically targeted. Catastrophizing patients are more likely to improve from self-instruction and verbal re-attribution, whereas non-catastrophisers benefit from interventions such as attention switching or distraction or attention control training (mindfulness). To reduce Catastrophizing, clinician should encourage the patient to recognize catastrophic cognitions and appreciate the unhelpful nature of these in coping with chronic pain as well as help them to develop alternative ways of thinking about it.

Generally, patients develop their own coping strategies to deal with their pain. The aim of coping strategy enhancement is to facilitate the implementation of appropriate active coping strategies, at the same time reducing the passive ways of coping. Before introducing new coping strategies, effectiveness of existing coping strategies should be analyzed. Use of particular strategies is often associated with patient's pain representations and pain control. If a patient believes that 
pills can alleviate pain, he may overuse medication and underuse the strategies related to psychosocial factors. Training in cognitively based coping strategies increases coping attempts, decreased negative thinking and lower tendency to report pain. Patients can test out which strategies are most effective for them. Coping strategy intervention can be targeted at medication use if there is evidence that medication is being used erratically or contrary to medical advice.

\section{Contingency Management}

Contingency management is aimed at reducing the frequency of reinforcement of pain behaviours and to increase the frequency of non-pain behaviours by systematically rewarding them. Therapeutic goals include promotion of increased physical activity, reduction in medication use, and prevention of unnecessary disability. Contingency management is indicated when there are no physical findings or patient's report of pain is clearly in excess to physical findings. These treatment strategies are less appropriate when physical findings account for patient's presentation and when there is no evidence of inactivity. It is important to address reinforcement of pain behaviours by family members and significant others by discussing these issues with them in an individual session. In addition to this, pacing of activities by using activity schedule is also very useful for patients who engage in burst of activity. The rationale for this strategy is 'do what you plan to do, not what you feel like to doing'. A graph with two axes (the ' $x$ ' axis for time and ' $y$ ' axis for amount of activity or pain intensity) can be used to make patients realize the fact that they are engaging in bursts of activities i.e. doing less on a 'good day' and more on a 'bad day', They are encouraged to sustain a similar level of activity irrespective of pain experience and increase level of activity gradually.

\section{The other behavioral elements in CBT may include}

- $\quad$ Setting up behavioral experiments to test irrational thoughts against reality

- $\quad$ Target setting and activity scheduling

- Teaching specific skills such as relaxation

- $\quad$ Role playing, behavioral rehearsal, therapist modeling coping behaviors

- $\quad$ Problem solving skills

\section{Relaxation Training}

Relaxation is defined several ways; as a psycho physiological state characterized by parasympathetic dominance of multiple visceral and somatic systems; the absence of physical, mental, and emotional tension; the opposite of Canon's fight-or-flight response. Relaxation is a learned skill with the potential to offset the negative effects of physical and psychological stress and rebalance the body, mind, and spirit. This technique has been observed to assist the individual to respond to life's challenges in more healthy ways, bringing involuntary responses such as heart rate, blood pressure, respiration, blood flow to muscles, muscle tension, and adrenalin secretion under voluntary control. Relaxation states allow the individual the opportunity to experience an inward focus of attention, an awareness of an altered perception of time and place, control of personal state of awareness, and a relaxed inner calmness or sense of a sacred healing space. 


\section{Relaxation Procedures}

\section{Jacobson's Progressive Muscles Relaxation (JPMR):}

JPMR is performed by first tensing, and then relaxing, the muscles of the body, one group at a time. Muscle groups can be divided a number of different ways, but a common method is to use the following groupings:

- Hands and arms

- $\quad$ head, neck, and shoulders;

- $\quad$ trunk, including chest, stomach and back;

- $\quad$ thighs, buttocks, legs, and feet.

The patient lays or sits in a comfortable position, and then starts with the first muscle group, focusing on the feeling of the muscles and the absence or presence of tension.

Release-only Relaxation: Like progressive relaxation, release-only relaxation focuses on relieving feelings of tension in the muscles. However, it eliminates the initial use of muscle tensing as practiced in progressive relaxation, focusing instead solely on muscle relaxation. Release-only relaxation is usually recommended as the next step in relaxation therapy after progressive relaxation has been mastered.

Deep Breathing Exercises: Deep breathing exercises are best performed while laying flat on the back, usually on the floor with a mat. The knees are bent, and the body is relaxed. One hand should be placed on the chest and one on the abdomen to monitor breathing technique. The individual takes a series of long, deep breaths through the nose, attempting to raise the abdomen instead of the chest. Air is exhaled through the relaxed mouth. Deep breathing can be continued for up to 20 minutes.

Cue-controlled Relaxation: Cue-controlled relaxation is an abbreviated tension-relief technique that combines elements of release-only relaxation and deep breathing exercises. It uses a cue, such as a word or mental image, to trigger immediate feelings of muscle relaxation. The cue must first be associated with relaxation in the individual's mind. This is accomplished by choosing the cue, and then using it in breathing and release-only relaxation exercises repeatedly until the cue starts to automatically trigger feelings of relaxation outside of the treatment sessions. Cues can be as simple as the word "relax", a visual cue, such as a mental image of a white sand sea beach, a flower-filled meadow.

Autogenic Training: Instead of focusing directly on muscle tension as a means to induce relaxation, autogenic training (AT) involves a set of exercises targeted at various physiological end-organs whereby a state of relaxation might be induced. These are based on the work of Schultz and further developed by Luther. "Autogenics", as a term, essentially means "selfexercise" or "self-induction" therapy and relies on a theoretical rationale whereby the client gains psychophysiological self-control of autonomic functioning and is able to achieve relaxation. 
Mindfulness Meditation: It refers to a family of techniques which have in common a conscious attempt to focus attention in a non-analytical way and an attempt not to dwell on discursive, ruminating thought. Such exercises vary widely and can involve sitting still and counting breaths, attending to a repeated thought, or focusing on virtually any simple external or internal stimulus. Vipassana or mindfulness meditation or therapeutic approaches based on it have received considerable attention in last two decades. In mindfulness meditation a 'choiceless' and non-judgmental awareness is achieved through practice of various kinds of procedures. The focus is to make both the mind and body relaxed. Clients are asked to pay attention to the incoming and outgoing breath, their thoughts, feelings and sensations. They are asked to be nonjudgmental about their thoughts and experience bodily sensations and feelings and all kinds of mental events as they occur naturally without any kind of regulation. Mindfulness meditation has been found to be very useful in patients with chronic pain.

\section{Hypnosis}

Hypnosis has been described as a sleep-like state that may be induced through motivation, relaxation, and concentration. The hypnotic induction process involves quieting muscles to enable one to attend mentally to positive statements and affirmations. The term hypnosis generally refers to the combination of suggestion and imagery that are employed to create an altered state of awareness and its practice has been helpful in chronic pain and other medical and surgical practices.

Notwithstanding the relatively benign nature of the relaxation procedures discussed, adverse effects have been reported, and precautions and contraindications have been detailed. Reported effects are generally transient and include: unwelcome and intrusive imagery, anxiety, panic, dizziness, confusion, restlessness, and headache.

\section{Biofeedback}

Biofeedback or applied psychophysiological feedback is a patient-guided treatment that teaches an individual self-regulation of bodily processes. This is achieved by detecting, amplifying, and displaying specific physiological process in such a way that the patient can be trained to voluntarily modify these processes. The name biofeedback refers to the biological signals that are fed back, or returned, to the patient in order for the patient to develop techniques of controlling them. During biofeedback, one or more special sensors are placed on the body. These sensors measure muscle tension, brain waves, heart rate, body temperature, and translate the information into a visual and/or audible readout, such as a paper tracing, a light display, or a series of beeps.

While the patient views the instantaneous feedback from the biofeedback monitors, he begins to recognize what thoughts, fears, and mental images influence his physical reactions. By monitoring this relationship between mind and body, he can then use thoughts and mental images deliberately to manipulate heartbeat, brain wave patterns, body temperature, and other bodily functions, and to reduce feelings of stress. This is achieved through relaxation exercises, mental imagery, and other cognitive therapy techniques. The patient is able to recognize the state 
of relaxation or visualization necessary to alleviate symptoms; the biofeedback equipment itself is no longer needed.

Biofeedback is commonly used to help in the treatment of pain. It can be used to identify and retrain body habits that produce or maintain pain. Biofeedback has been shown to be effective in the management of migraine headaches, fibromyalgia, temporomandibular disorders, and rheumatoid arthritis, Raynaud's disease, tension headaches, headaches in children and the pain associated with irritable bowel syndrome.

\section{SUMMARY AND CONCLUSIONS}

CBT, relaxation training and biofeedback has proven to be effective in reducing pain and disability when it is used as part of a therapeutic strategy for chronic pain. CBT addresses the psychological component of pain, including attitudes and feelings, coping skills, and a sense of control over one's condition. It can provide educational information and diffuse feelings of fear and helplessness. It can help a patient look at ways in which their attitudes contribute to inaccurate and unrealistic expectations, and can help them find a more realistic and balanced view of the problem. Relaxation approaches and biofeedback can help people in chronic pain lower their overall level of arousal, decrease muscle tension, control distress, and decrease pain, depression and disability. Incorporating biopsychosocial model in pain the management of pain not only improves pain symptoms but also leads to speedy recovery and well-being of patients.

\section{REFERENCES}

Banks, S.M., \& Kerns, R.D (1996).Explaining high rates of depression in chronic pain: a diathesis-stress framework. Psychological Bulletin 119, 95-110.

Beck, A. T. (1979). Cognitive therapy of depression. New York, NY:Guilford Press.

Bonica J.J. The need of taxonomy (1979). Pain. 6 (3), 247-248

Dember, W. N. (1974). Motivation and the cognitive revolution. American Psychologist, 29 (3), $161-168$.

Donello, J.E.; Guan, Y.; Tian, M.; Cheevers, C.V.; Alcantara, M.; Cabrera, S.; Raja, S.N. \& Gil, D.W. (2011) A peripheral adrenoceptor-mediated sympathetic mechanism can transform stress-induced analgesia into hyperalgesia. Anesthesiology, 114 (6): 14031416.

Eccleston, C., Williams, A. C., \& Morley, S. (2009). Psychological therapies for the management of chronic pain (excluding headache) in adults. Cochrane Database of Systematic Reviews, 2009(2), Article No. CD007407.

Engel G.L (1977). The need for a new medical model: a challenge for biomedicine. Science 196, 129-136.

Fordyce, W. E., Fowler, R. S., \& DeLateur, B. (1968). An application of behavior modification technique to a problem of chronic pain. Behav iour Research and Therapy, $6(1), 105-107$. 
Gamsa, A. (1994). The role of psychological factors in chronic pain: A half century of study. Pain, 57 (1), 5-15.

Hooper, R. (1811) Quincy's Lexicon-Medicum. A New Medical Dictionary. London.

Keefe, F. J., Shelby, R. A., Somers, T. J., Varia, I., Blazing, M., Waters,S. J., Bradley, L. (2011). Effects of coping skills training and sertraline in patients with non-cardiac chest pain: A randomized controlled study. Pain, 152 (4), 730-741.

Kerns, R. D., Rosenberg, R., \& Jacob, M. C. (1994). Anger expression and chronic pain. Journal of Behavioral Medicine, 17, 57-67.

Lazarus, R. S., \& Folkman, S. (1984). Stress, appraisal, and coping. New York, NY: Springer.

Luciano, J.V.; Martínez, N.; Peñarrubia-María, M.T.; Fernández-Vergel, R.; García-Campayo,J.; Verduras, C.; Blanco, M.E.; Jiménez, M.; Ruiz, J.M.; López del Hoyo, Y.; SerranoBlanco, A. \& Fibro QoL Study Group. (2011) Effectiveness of a psychoeducational treatment program implemented in eneral practice for fibromyalgia patients: a randomized controlled trial. Clinical Journal of Pain, 27(5), 383-391.

Meichenbaum, D. (1976). Cognitive factors in biofeedback therapy. Biofeedback SelfRegulation, 1 (2), 201-216.

Montgomery, G.H. \& Kirsch, I. (1997) Classical conditioning and the placebo effect. Pain,72 (12): 107-113.

Schmidt, S., Grossman, P., Schwarzer, B., Jena, S., Naumann, J., \& Walach, H. (2011). Treating fibromyalgia with mindfulness-based stress reduction: Results from a 3-armed randomized controlled trial. Pain, 152 (2), 361-369.

Turk, D. C., Meichenbaum, D., \& Genest, M. (1983). Pain and behavioral medicine: A cognitive-behavioral perspective. New York, NY: Guilford Press.

Turk.D.C. (1995). Viewpoint: Treatment of chronic pain: Clinical outcomes, costeffectiveness \& cost benefits. www.medscape.com/viewarticle/410012

Weintraub, M.I. (2003) Complementary and alternative methods of treatment of neck pain. Physical Medicine \& Rehabilitation Clinic of North America, 14 (3), 659-674. 